\title{
Ensembles of nanoelectrodes modified with gold nanoparticles: characterization and application to DNA-hybridization detection
}

\author{
Morena Silvestrini • Paolo Ugo
}

Received: 20 June 2012 /Revised: 31 July 2012 /Accepted: 13 August 2012 / Published online: 6 September 2012

(C) Springer-Verlag 2012

\begin{abstract}
A new method to increase the active area $\left(A_{\text {act }}\right)$ of nanoelectrode ensembles (NEEs) is described. To this aim, gold nanoparticles (AuNPs) are immobilized onto the surface of NEEs using cysteamine as a cross-linker able to bind the AuNPs to the heads of the nanoelectrodes to obtain the so-called AuNPs-NEEs. The analysis of the cyclic voltammograms recorded in pure supporting electrolyte showed that the presence of the nanoparticles reflects in an, approximately, ten-times increase in the electrochemically active area of the ensemble. The measurement of the amount of electroactive polyoxometalates, which can be adsorbed on the gold surface of NEEs vs. AuNPs-NEEs, confirmed a significant increase of active area for the latter. These evidences indicate that there is a good electronic connection between the AuNPs and the underlying nanoelectrodes. The possibility to exploit AuNPs-NEEs for biosensing application was tested for the case of DNAhybridization detection. After immobilization on the gold surface of AuNPs-NEEs of a thiolated singlestranded DNA, the hybridization with complementary sequences labeled with glucose oxidase (GOx) was performed. The detection of the hybridization was achieved by adding to the electrolyte solution the GOx substrate (i.e., glucose) and a suitable redox mediator, namely the (ferrocenylmethyl) trimethylammonium $\left(\mathrm{FA}^{+}\right)$cation; when the hybridization occurs, an electrocatalytic increase of the oxidation current of $\mathrm{FA}^{+}$is recorded.
\end{abstract}

Published in the special issue Analytical Science in Italy with guest editor Aldo Roda.

M. Silvestrini $\cdot$ P. Ugo $(\bowtie)$

Department of Molecular Sciences and Nanosystems, University

Ca' Foscari of Venice,

Santa Marta 2137,

30123 Venice, Italy

e-mail: ugo@unive.it
Comparison of electrocatalytic current recorded at DNA modified NEEs and AuNPs-NEEs indicate, for the latter, a significant increase in sensitivity in the detection of the DNA-hybridization event.

Keywords Nanoelectrode $\cdot$ Ensembles · Gold nanoparticles · DNA hybridization · Voltammetry

\section{Introduction}

Nanoelectrode ensembles (NEEs) are electrochemical nanodevices fabricated by the electroless or electrochemical deposition of metal fibers within the pores of a templating membrane [1], typically, track-etched polycarbonate membranes. The final assembly is a $2 \mathrm{D}$ composite device (herein called 2D NEE), constituted by inlaid nanodisc electrodes embedded in the insulating polymer.

2D NEEs present some unique characteristics, such as highly improved signal-to-background current ratio, very low detection limits, high sensitivity to charge transfer kinetics $[2,3]$; moreover, they are suitable to extreme miniaturization. However, 2D NEEs show some limits in all those applications where systems with high electroactive area are required, for instance, in the case of electrochemical devices and sensors based on the use of electroactive molecules or redox mediators immobilized on electrode surfaces.

Recently, it was shown that 3D ensembles of metal nanowires can be obtained by plasma [4] or chemical etching [5] of 2D NEE. Such 3D NEEs have been successfully applied to prepare advanced electrochemical sensors [6, 7]. However, the etching causes an increase of the capacitive doublelayer charging current and a consequent decrease of the signal/background current ratio [7], so losing one of the advantages of NEEs. 
In the present work, a new strategy to fabricate 3D nanostructures on NEEs is proposed with the goal of increasing the nanostructured metal surface. A way to achieve this goal is based on the immobilization of gold nanoparticles (AuNPs) on the gold surface of the nanoelectrodes of a NEE by exploiting the binding properties of bifunctional thiols [8]. The gold surface is then functionalized by molecular recognition elements keeping as unaltered as possible the characteristics and analytical advantages of 2D NEEs. In such a way, it is possible to prepare electrochemical biosensors with improved capabilities as far as the high loading of biorecognition molecules and analytical sensitivity are concerned.

The analytical usefulness of such AuNPs-NEEs is demonstrated by exploiting the binding capabilities of thiolated oligonucleotides [9] to immobilize single-stranded DNA (ssDNA) probes on the AuNPs deposited on the NEE. The hybridization with complementary target strands is detected electrochemically using glucose oxidase (GOx) as the enzyme label bound to the target sequences and a ferrocene derivative as redox mediator [10].

The obtained experimental results constitute a proof of concept of the feasibility of a NEE-based electrochemical system with improved bioelectrochemical detection properties.

\section{Experimental}

Materials and apparatus

Trisodium citrate dihydrate was from Merck; cysteamine hydrochloride, phosphomolybdic acid, $\mathrm{H}_{3} \mathrm{PMo}_{12} \mathrm{O}_{40}$ (PMA), and glucose oxidase (GOx) from Aspergillus niger and the cross-linker sulfosuccinimidyl 4-[N-maleimidomethyl]-cyclohexane-1-carboxylate (sulfoSMCC) were all purchased from Sigma Aldrich. The salt (ferrocenylmethyl) trimethylammonium hexafluorophosphate $\left(\mathrm{FA}^{+} \mathrm{PF}_{6}{ }^{-}\right)$was prepared by metathesis of the (ferrocenylmethyl) trimethylammonium iodide (Alfa Aesar) with potassium hexafluorophosphate $99 \%$ (Alfa Aesar). Dithiothreitol (DTT) was from AppliChem. All other reagents were of analytical grade and were used as received. Purified water was obtained using a MilliRo plus Milli-Q (Millipore) water purification system.

Chloroauric acid was prepared dissolving an amount of metallic gold in aqua regia, heating the acidic solution under stirring and concentrating it by evaporation. Afterward, three subsequent additions of $1.2 \mathrm{M} \mathrm{HCl}$ and two additions of water were done. The solution was concentrated every time. The gold plating solution (sodium gold sulfite, $\left.\mathrm{Na}_{3}\left[\mathrm{Au}\left(\mathrm{SO}_{3}\right)_{2}\right]\right)$ was easily prepared starting with the reaction of chloroauric acid and a slight excess of sodium bicarbonate. This reaction allows the formation of sodium chloroaurate $(\mathrm{Na}$ $\left.\left(\mathrm{AuCl}_{4}\right)\right)$. The solution was allowed to concentrate until orange crystals were formed. At this point, the procedure reported by Abys et al. [11] was followed, adapting the various concentrations at the initial concentration of metallic gold used by us for the synthesis of chloroauric acid.

The chloroaurate crystals were dissolved in water, heated at $80{ }^{\circ} \mathrm{C}$ under stirring, and then barium hydroxide was added to the stirred solution, causing a color change to brown. Subsequently, $\mathrm{NaOH}$, preferably as concentrated aqueous solution, was added and the temperature was increased to boiling. The $\mathrm{pH}$ of this solution should be between 6 and 8. After adjusting of $\mathrm{pH}$, the solution was cooled and filtered. The precipitate was repeatedly rinsed with small amounts of cold water. Then, the precipitate was dissolved in water and the solution was heated at $50{ }^{\circ} \mathrm{C}$ under stirring. The solution was then cooled and the precipitate was collected. Heating, cooling, and filtration were repeated two times.

The final precipitate was dissolved in water, brought to $60-65{ }^{\circ} \mathrm{C}$, and subsequently, $\mathrm{Na}_{2} \mathrm{SO}_{3}$ was added under stirring. The solution was kept at this temperature until a purple precipitate was formed with disappearance of the previous brown precipitate. The solution was finally filtrated and kept in the dark until use.

Thiolated ssDNA (SHD1) and complementary thiolated ssDNA (SHD2) were purchased from Sigma Aldrich and had the following sequences:

\section{SHD1: HS-5' GTGGAAAGTGGCAATCGTGAAG 3' \\ SHD2: HS-5' TTTTTTCTTCACGATTGCCACTTT} CCAC 3'

All oligonucleotides stock solutions $(100 \mu \mathrm{M})$ were prepared in TE buffer $(50 \mathrm{mM}$ Tris, $100 \mathrm{mM} \mathrm{NaCl}, 1 \mathrm{mM}$ EDTA in $\mathrm{pH}$ 7.4) and kept frozen until use.

NAPTM-5 and NAPTM-10 columns prepacked with Sephadex ${ }^{\mathrm{TM}}$ G-25 DNA Grade were from GE Healthcare. Ultrafiltration devices (Vivaspin6 $30000 \mathrm{MWCO}$ ) were purchased from VWR.

The purification of the DNA-enzyme conjugate was carried out by fast protein liquid chromatography (FPLC; GE Healthcare, Äkta Explorer) using a MonoQ 5/50 GL column. Track-etched polycarbonate (PC) membrane filters were obtained from SPI-pore ${ }^{\mathrm{TM}}$ with nominal pore diameter of $30 \mathrm{~nm}$, average pore density of $6 \times 10^{8}$ pores $\mathrm{cm}^{-2}$, and coated with polyvinylpyrrolidone by the producer.

All electrochemical measurements were carried out at room temperature with a $\mathrm{CH} 660 \mathrm{~A}$ potentiostat controlled via personal computer by its own software, using a three-electrode single-compartment cell equipped with a platinum counter electrode and an $\mathrm{Ag} / \mathrm{AgCl}(\mathrm{KCl}$ 
saturated) reference electrode, to which all reported potential values are referred.

\section{Synthesis of ssDNA conjugated with GOx (D2-GOx)}

The complementary strand (SHD2) was conjugated with glucose oxidase (GOx) using sulfoSMCC methodology as previously described [12]. Briefly, $100 \mu \mathrm{L}$ of a 100 $\mu \mathrm{M}$ SHD2 solution was incubated with DTT $(60 \mu \mathrm{L}$ of a 1 M DTT) overnight at $37{ }^{\circ} \mathrm{C}$ to allow for the reduction of disulfide bonds. Before coupling, reduced SHD2 was purified by use of NAP5 and NAP10 size exclusion column. GOx was firstly desalted through a short-size exclusion column (NAP5) and then $400 \mu \mathrm{L}$ of the final solution $(60 \mu \mathrm{M}$ ca.) was added to $100 \mu \mathrm{L}$ of $2 \mathrm{mg}$ sulfoSMCC in dimethylformamide. The activation reaction was allowed to proceed for $1 \mathrm{~h}$ in dark at RT, and during this step, sulfoSMCC is coupled to the GOx surface through reaction with lysine amino groups. After the reaction completion, activated GOx was purified by NAP5 and NAP10 column using 0.01 M PBS pH 7.4 as elution buffer. The buffer solutions of SHD2 and activated GOx were immediately combined and incubated in the dark for $3 \mathrm{~h}$ at RT. After incubation, the buffer was exchanged to $20 \mathrm{mM}$ Tris $\mathrm{pH} 8.2$ using an ultrafiltration device (Vivaspin6 30000 MWCO) and D2GOx purified using FPLC system with an ion exchange column. The fractions containing the conjugate were collected, concentrated, and redissolved in $0.01 \mathrm{M}$ PBS, pH 7.4.

Synthesis and characterization of gold nanoparticles (AuNPs)

AuNPs were synthesized by reduction of tetrachloroauric acid by trisodium citrate following the method described by Gooding et al. [13]. The final colloidal gold solution was kept in the dark until use. The obtained AuNPs were characterized by UV-vis spectrophotometric measurements performed on the obtained colloidal gold solution. In the latter case, the average diameter of the AuNPs was calculated by using the equation proposed by Haiss et al. [14],

$d=\exp \left(B_{1} A_{\mathrm{spr}} / A_{450}-B_{2}\right)$
Fig. 1 Scheme of an Au-NEE prepared using a track-etched polycarbonate membrane as template. (A) Particular of the section of the active area; (B) top view and (C) section of the all NEE ready for use as working electrode. (a) Track-etched golden membrane. (b) Copper adhesive tape with conductive glue to connect to instrumentation. (c) Aluminum adhesive foil with non-conductive glue. (d) Insulating tape. Not all details (namely, the nanoelectrodes dimension) are in the scale
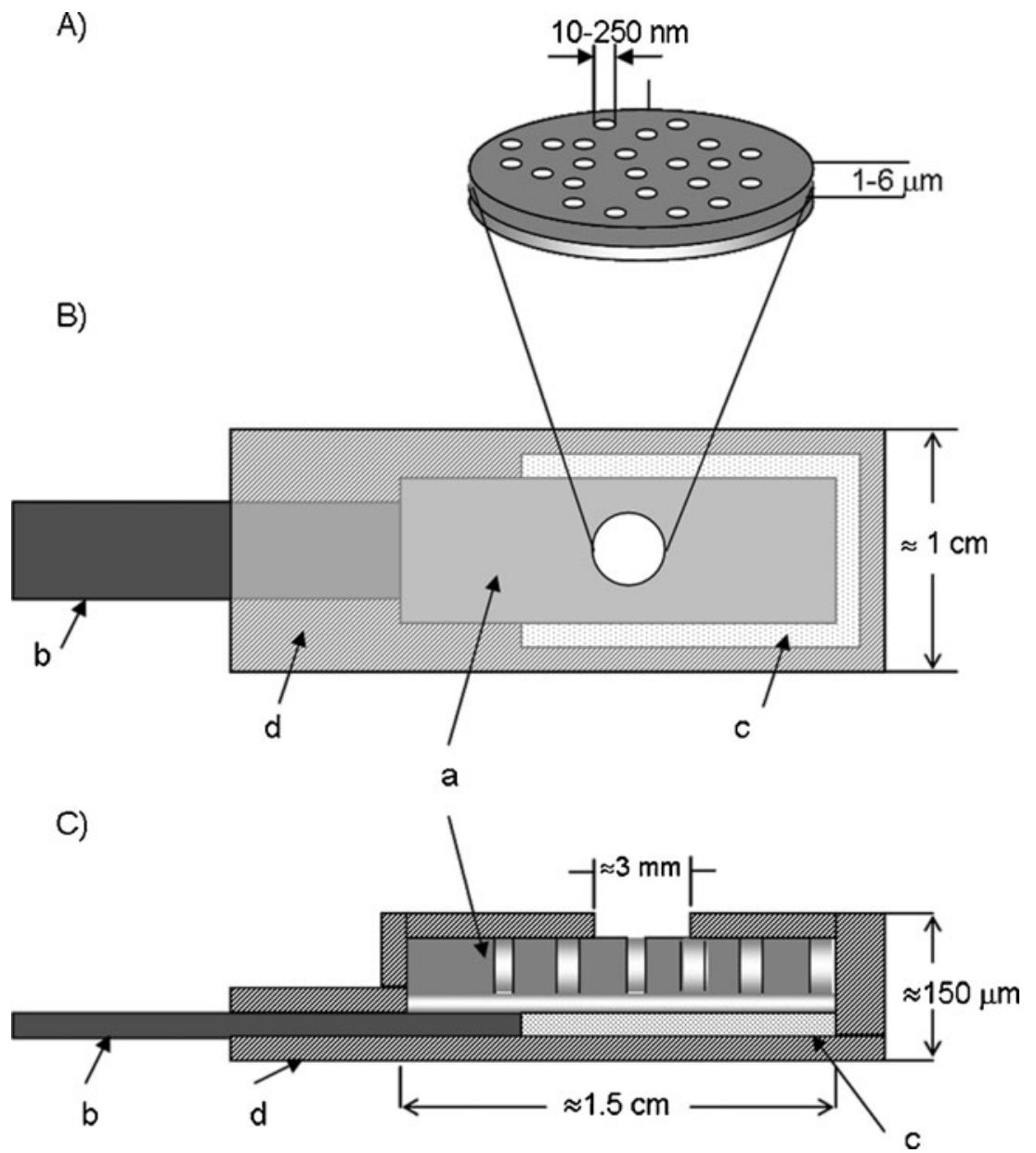
Fig. 2 Design of a DNA

hybridization sensor based on

AuNPs-NEE assembly. (a)

Modification of NEE with cys-

teamine. (b) Immobilization of

gold nanoparticles. (c) Func-

tionalization with probe

sequences (SHD1) and subse-

quent hybridization with com-

plementary target conjugated

with GOx (D2-GOx). Note: the

dimensions are not in the scale

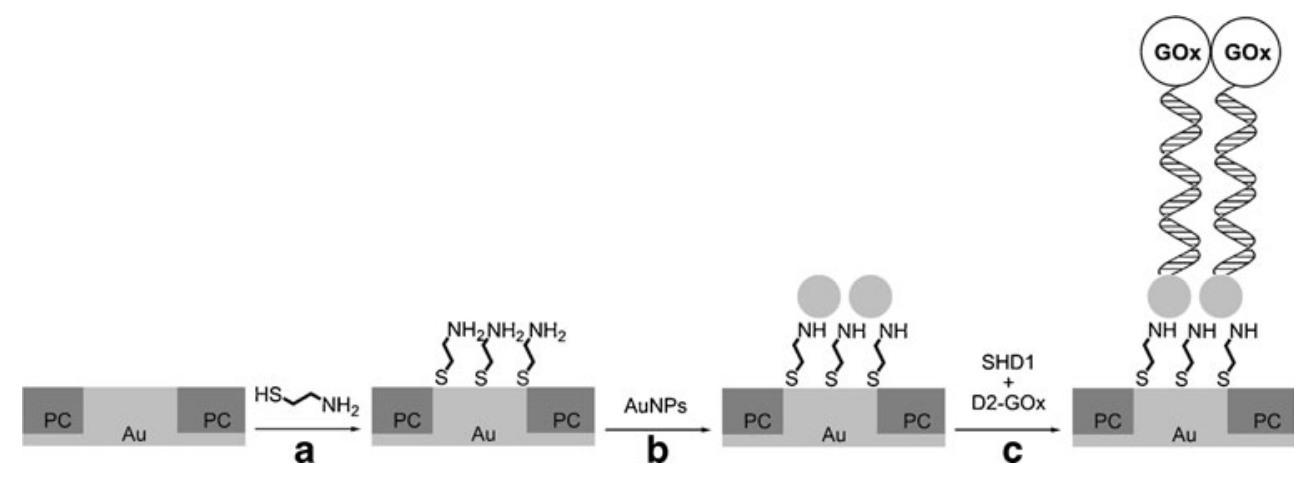

Fig. 3 (a) Comparison

between the cyclic

voltammograms recorded in

$1 \mathrm{mM} \mathrm{KNO}_{3}$ at a bare NEE (full

line), with those obtained with a not thiolated NEE (dotted line) and a cyst-NEE (dashed line), both immersed for $8 \mathrm{~h}$ into the colloidal gold solution. Scan rate is $50 \mathrm{mVs}^{-1}$. Note the different scales on the $y$-axis. (b)

Plots of the charging current

(Ic) vs. scan rate

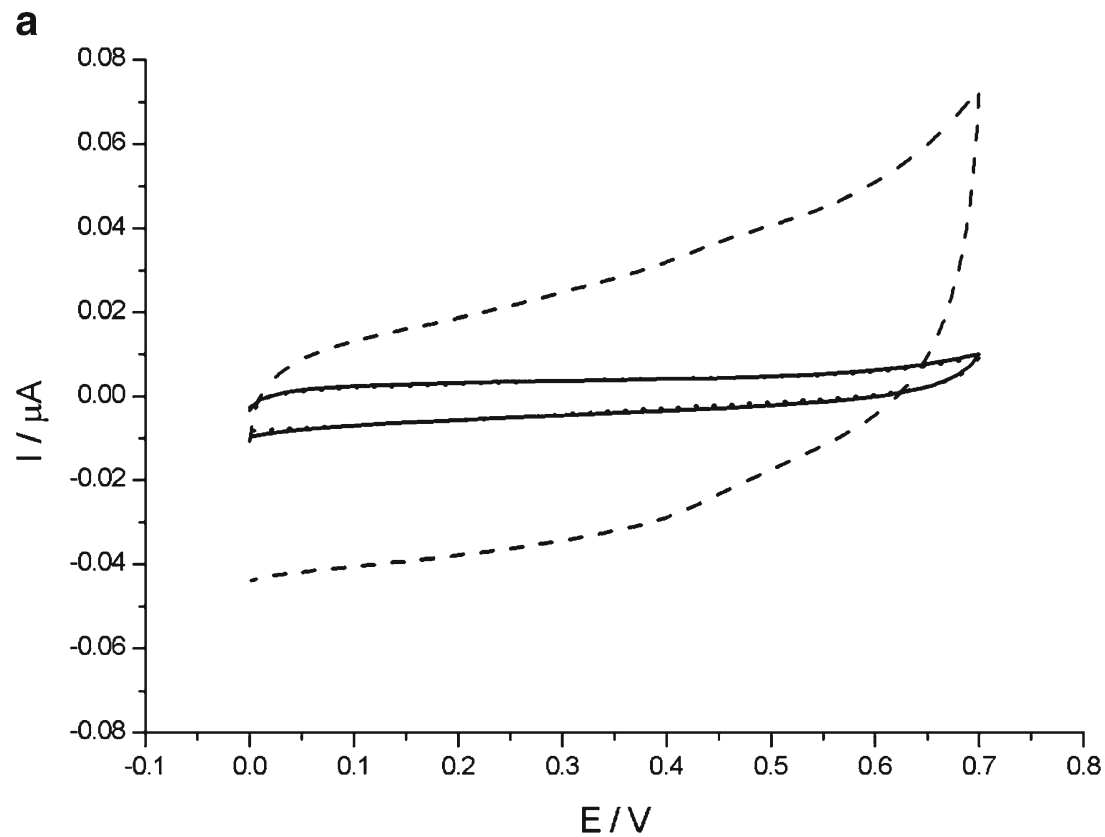

b

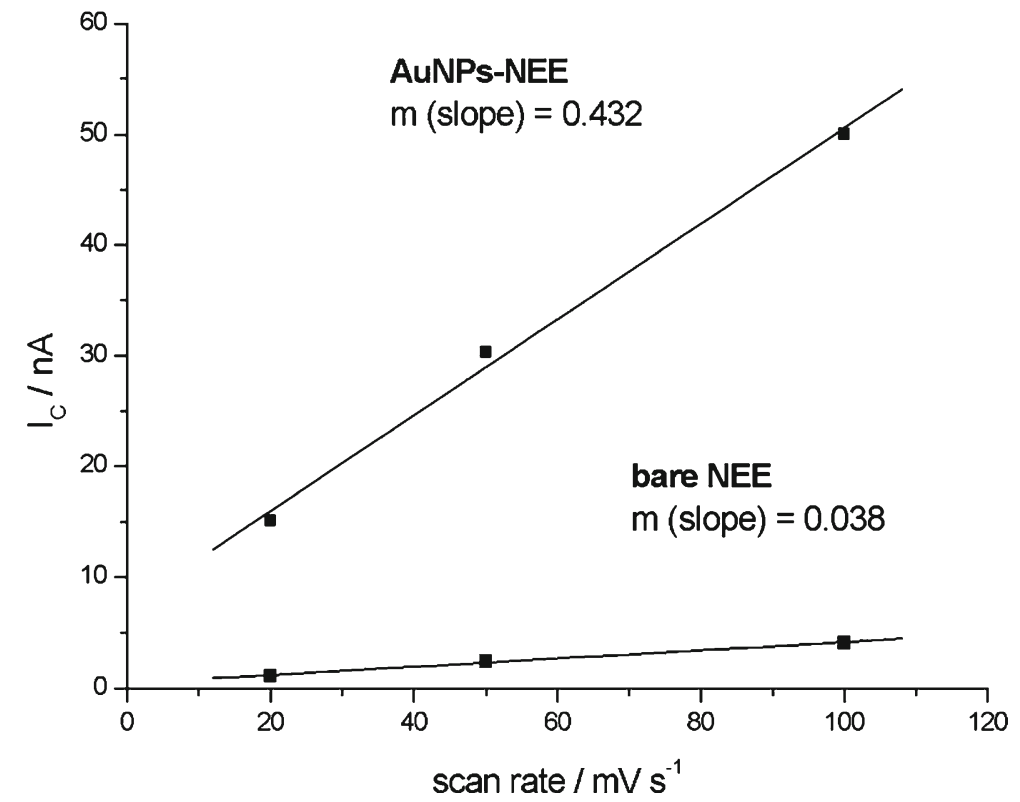


where $B_{1}=3.00, B_{2}=2.20$ and $\mathrm{A}_{\mathrm{spr}}$ and $\mathrm{A}_{450}$ are the absorbance at the surface plasma resonance peak and at $450 \mathrm{~nm}$, respectively. In our case, $d$ resulted equal to $16 \pm 2 \mathrm{~nm}$.

This value agrees quite well with data obtained by transmission electron microscopy (not shown) which confirmed the presence of spherical nanoparticles with an average diameter of approximately $20 \mathrm{~nm}$.

Electrode preparation and functionalization

\section{Fabrication of NEEs and modification with AuNPS}

Growth of the nanoelectrodes in track-etched polycarbonate membranes was performed according to the electroless procedure proposed by Menon and Martin [1] and following modifications [15].

2D NEEs were assembled, according to the scheme shown in Fig. 1, from pieces of golden membrane, as previously described [16]. The final geometric area $\left(A_{\text {geom }}\right)$ of the NEEs is defined as the overall area of the ensemble (templating polycarbonate + nanoelectrodes) exposed to the electrolyte solution [1]. As in Fig. 1, $A_{\text {geom }}$ is determined by the diameter of a hole punched in the outer insulating tape (component (d) in Fig. 1). The diameter of the hole typically used in our lab is $0.3 \mathrm{~cm}$, so that $A_{\text {geom }}$ for the NEEs used here is $0.07 \mathrm{~cm}^{2}$.

AuNPs-NEEs were prepared exploiting interactions between the gold surface (the metal surface of both NPs and nanodisc electrodes) and specific functional groups $\left(-\mathrm{SH},-\mathrm{NH}_{2}\right)$ of cysteamine $[17,18]$.

For the modification of NEE with cysteamine, the electrode was dipped overnight ( $16 \mathrm{~h} \mathrm{ca}$.), into a $10 \mathrm{mM}$ cysteamine solution in water. Preliminary experiments showed that this treatment performed on NEEs does not change their electrochemical performances. Cysteamine is to be preferred to dithiols because it cannot form multilayers or disulfide bridges [13]. Previous studies showed that SH terminal groups bind preferentially to gold surfaces leaving the amino groups available for the subsequent NPs immobilization [13]. The formation of a 3D structure takes place through the attachment of the nanoparticles to the free amino functionalities present on the monolayers, by weak covalent bonds $[19,20]$.

The NEE modified with SAMs (cyst-NEE) was then immersed into the colloidal gold solution, prepared as described before. The incubation was allowed to proceed for $8 \mathrm{~h}$ at RT.

In order to ascertain whether the presence of SAMs effectively favors the immobilization of NPs, comparison samples were prepared by incubating a not thiolated NEE into the NPs colloidal dispersion for $8 \mathrm{~h}$.

\section{Functionalization of AuNPs-NEEs with oligonucleotides}

Five microliters of a $2 \mu \mathrm{M}$ SHD1 solution in $10 \mathrm{mM}$ TE buffer $(\mathrm{pH}=7.2)$ with $1 \mathrm{M} \mathrm{NaCl}$ was spotted onto the geometric area of an AuNPs-NEE at RT. Note that the electrostatic repulsion between oligonucleotides is minimized [21] and a high coverage of the electrodic surface can be achieved by operating at high ionic strength.

After $1 \mathrm{~h}$, the electrode was accurately rinsed with TE buffer and subsequently spotted with $5 \mu \mathrm{L}$ of $2 \mu \mathrm{M}$ target conjugated with GOx (D2-GOx) in $10 \mathrm{mM}$ TE buffer $(\mathrm{pH}=7.2)$ with $1 \mathrm{M} \mathrm{NaCl}$. Also in this case, after $1 \mathrm{~h}$ of incubation, the NEE was rinsed by immersion in TE buffer for ten more minutes and allowed to dry in air. The same functionalization steps were performed, for comparison, on a bare NEE. The overall functionalization scheme is summarized in Fig. 2.

\section{Results and discussion}

\section{Electrochemical characterization of AuNPs-NEEs}

Figure 3a compares the cyclic voltammograms recorded at a bare NEE (full line), with those obtained with a not thiolated NEE (dotted line) and a cyst-NEE (dashed line), both immersed for $8 \mathrm{~h}$ into the colloidal gold solution. The characterization was performed in pure supporting electrolyte $\left(1 \mathrm{mM} \mathrm{KNO}_{3}\right)$ at $50 \mathrm{mVs}^{-1}$. Signals recorded in these experimental conditions correspond indeed to the double layer charging current, given as [22]:

$I_{\mathrm{C}}=v C_{\mathrm{dl}} A_{\mathrm{act}}$

where $v$ is the voltammetric scan rate, $C_{\mathrm{d} 1}$ is the double layer capacitance of the metal/electrolyte interface and

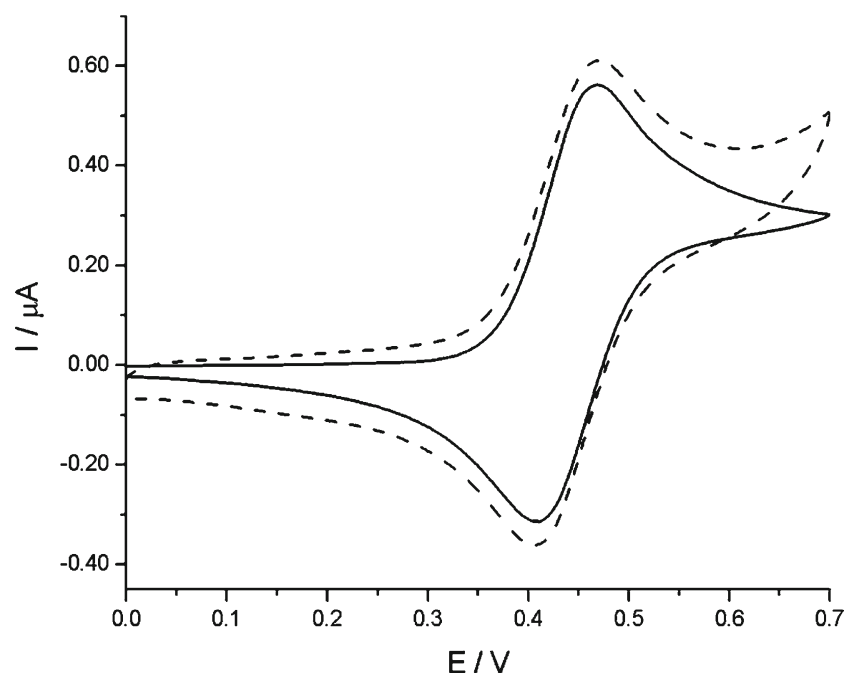

Fig. 4 Cyclic voltammograms recorded with a bare NEE (full line) and an AuNPs-NEE (dashed line) in $10 \mathrm{mM}$ PBS (pH 7.0) containing $0.1 \mathrm{mM} \mathrm{FA}^{+} \mathrm{PF}_{6}^{-}$. Scan rate is $10 \mathrm{mVs}^{-1}$ 
$A_{\text {act }}$ is the active area (i.e., the metal surface of the nanoelectrodes exposed to the electrolyte solution) of the NEE. $I_{\mathrm{C}}$ was evaluated from the $\mathrm{CVs}$ in Fig. 3a as $1 / 2 \Delta I$ at $400 \mathrm{mV}$, where $\Delta I$ is the difference in current recorded during the forward and backward scan [22].

The comparison between the full and dotted-line CVs in Fig. 3a indicates that in the absence of cysteamine, the double-layer charging current is not influenced by the treatment with the NPs since the nanoparticles cannot bind to the gold surfaces of the NEE. On the other hand, the CV for the cyst-NEE treated with AuNPs (dashed line) shows a dramatic increase in capacitive current, which can be attributed to a significant increase in active area related to the binding of the AuNPs onto the NEE.

As shown in Fig. 3b, $I_{\mathrm{C}}$ scales linearly with $v$ both for the bare NEE and for the cyst-NEE treated with AuNPs, with a significant increase of slope for the latter case.

In principle, the analysis of the background $\mathrm{CV}$ s recorded at different scan rates could allow one to calculate $A_{\text {act }}$ by applying Eq. (2) [16]. However, performing the exact calculation here would require the quantification of the change in $C_{\mathrm{dl}}$ caused by the modification of the NEE with cysteamine and AuNPs, which is indeed unknown. Note that the AuNPs are citrate-coated so that their double layer capacitance can be different from the one of a bare gold surface.
Fig. 5 Cyclic voltammograms recorded in $0.5 \mathrm{M} \mathrm{H}_{2} \mathrm{SO}_{4}$ with (a) a bare NEE and (b) an AuNPs-NEE both treated with PMA. Scan rates are 5, 10, 20, 50 , and $100 \mathrm{mVs}^{-1}$

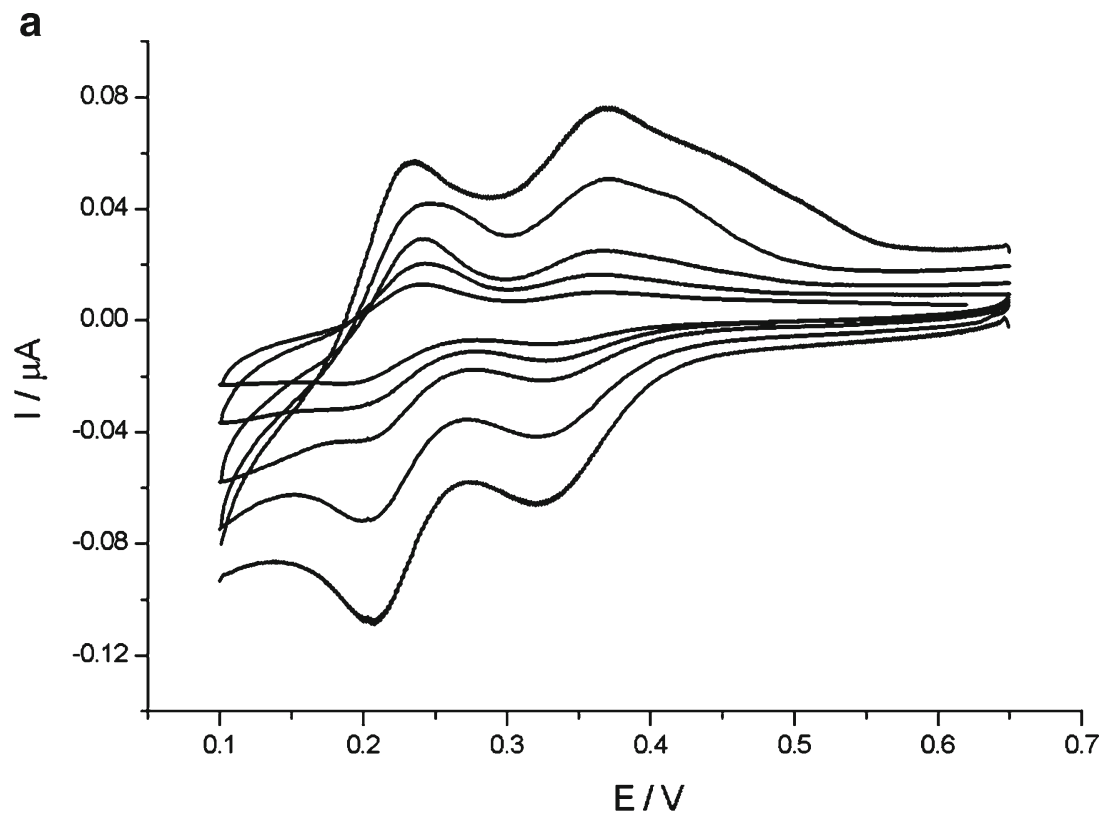

b

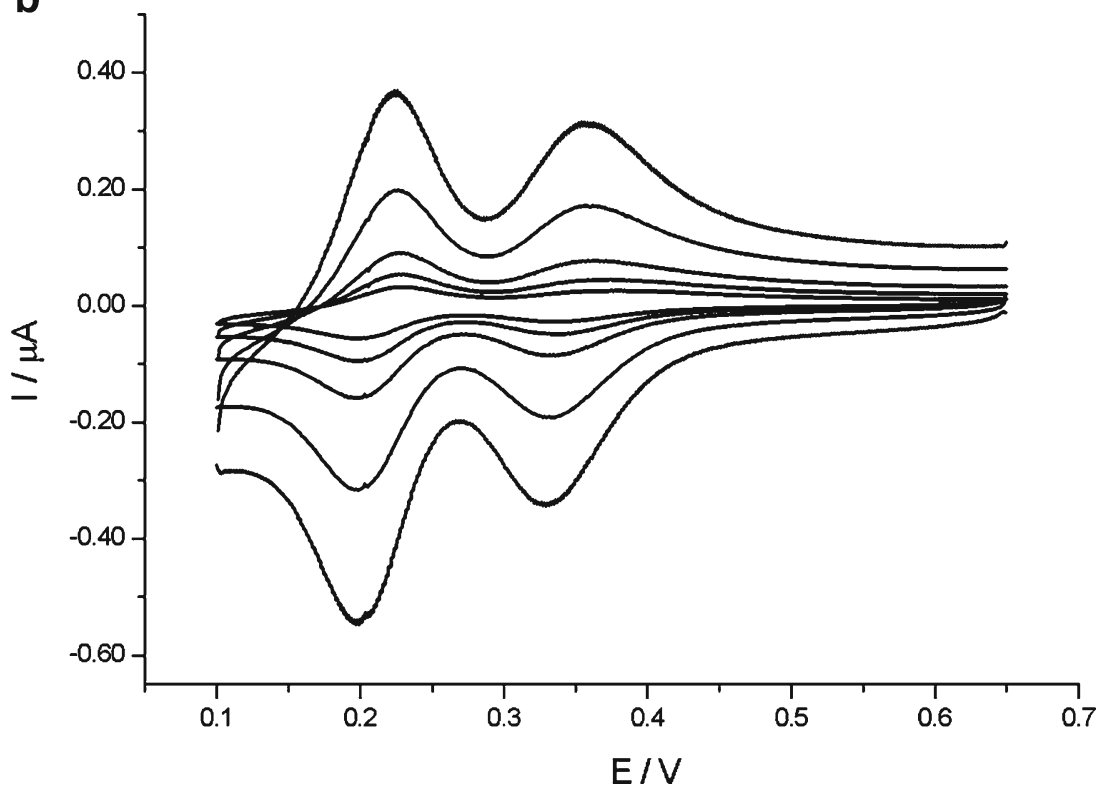


This notwithstanding, the results presented in Fig. 3 demonstrate, unequivocally, that a significant increase in active area is caused by the treatment of the NEE with cysteamine and AuNPs.

Figure 4 shows the CVs recorded both at a bare NEE and at an AuNPs-NEE in $0.1 \mathrm{mM} \mathrm{FA}^{+}, 10 \mathrm{mM}$ PBS (pH 7.0) at $10 \mathrm{mVs}^{-1}$, which is in the presence of a soluble redox probe of well-known electrochemical behavior, namely the $\mathrm{FA}^{+}$ cation [1]. These CVs put in evidence that, in the presence of the AuNPs, the active area increases; consequently, the capacitive current increases (dashed curve). However, the reversible oxidation of $\mathrm{FA}^{+}$is still well resolved and its oxidation peak current is comparable to the one recorded with the bare unmodified NEE. The fact that the net Faradaic peak current does not increase with the deposition of the AuNPs, while the capacitive current increases, is related to the fact that NEEs (even AuNPs-NEEs) operate in total overlap diffusion regime [16]; under such condition, $I_{\mathrm{p}}$ (peak current) depends on $A_{\text {geom }}$ while $I_{\mathrm{C}}$ depends on $A_{\text {act }}$ $[1,7]$. Note that with the deposition of the AuNPs, $A_{\text {geom }}$ does not change.

Data in Fig. 4 demonstrate that AuNPs immobilization does not block the electrochemistry of the NEEs and that AuNPs are electrically connected with the nanoelectrodes.

A further characterization was made in order to quantify the increase in active area and change in roughness, when passing from a $2 \mathrm{D}$ NEE to a more complex $3 \mathrm{D}$ structure.

To this aim, both a bare NEE and AuNPs-NEE were treated with a probe, namely phosphomolybdic acid (PMA), which is electroactive and which adsorbs on gold surfaces, forming a monolayer [23]. To achieve this, both the NEE and AuNPs-NEE were immersed for $1 \mathrm{~h}$ at RT in a $5 \mathrm{mM}$ PMA solution. Subsequently, the electrodes were rinsed with water and characterized by cyclic voltammetry in $0.5 \mathrm{M} \mathrm{H}_{2} \mathrm{SO}_{4}$ [7].

Figure 5 reports a comparison between the $\mathrm{CVs}$ recorded at different scan rates at a bare NEE (Fig. 5a) and at an AuNPs-NEE (Fig. 5b), both treated with PMA and transferred into pure supporting electrolyte. The voltammograms show two reversible processes, which correspond to the following consecutive reduction steps:

$$
\begin{aligned}
& \mathrm{PMo}_{12} \mathrm{O}_{40}{ }^{3-}+2 \mathrm{e}^{-} \rightarrow \mathrm{PMo}_{12} \mathrm{O}_{40}{ }^{5-} \\
& \mathrm{PMo}_{12} \mathrm{O}_{40}{ }^{5-}+2 \mathrm{e}^{-} \rightarrow \mathrm{PMo}_{12} \mathrm{O}_{40}{ }^{7-}
\end{aligned}
$$

In both cases, the peak current values depend linearly on the scan rate, indicating a surface process related to molecules adsorbed on the gold surface of the NEE.

The electrochemical signal of the PMA is significantly higher at the AuNPs-NEE (Fig. 5b) in agreement with the higher active area due to the presence of the nanoparticles.

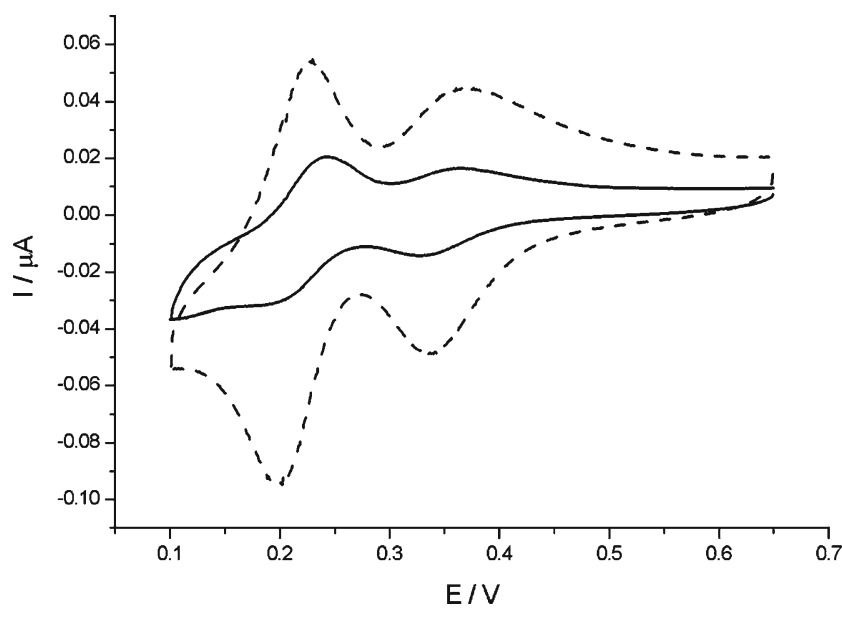

Fig. 6 Cyclic voltammograms recorded in $0.5 \mathrm{M} \mathrm{H}_{2} \mathrm{SO}_{4}$ with a bare NEE (full line) and an AuNPs-NEE (dashed line), both treated with PMA. Scan rate is $10 \mathrm{mVs}^{-1}$

Figure 6 shows the comparison between the voltammograms relative to both NEEs recorded at $10 \mathrm{mVs}^{-1}$.

By integrating the first reduction peak, it is possible to obtain the charge value $(Q)$ associated with the peak. From this value, the number of moles adsorbed $(m)$ onto both NEEs was calculated, by the following equation:

$m=Q / n F$

where $n$ is the number of electrons exchanged in the reaction (in this case two) and $F$ is the Faraday constant. By the Avogadro's number, one can also calculate the total number of molecules adsorbed on both electrodes. Relevant results are listed in Table 1.

Both charge and number of moles are larger for the AuNPs-NEE; this confirms the possibility to immobilize larger amounts of electroactive molecules (almost four times higher) onto the AuNPs-NEE, with respect to a "conventional" 2D NEE.

PMA is a Keggin-type heteropoly anion characterized by a spherical structure with size of about $1 \mathrm{~nm}$, which adsorbs through the formation of a monolayer [24-26]. For simplification, we considered this polyoxometalate as a sphere with $d=1 \mathrm{~nm}$, therefore with a cross area of approximately $0.78 \times 10^{-14} \mathrm{~cm}^{2}$. From suitable calculation, the surface of the ensemble coated by the PMA molecules results to $2.3 \times$

Table 1 Values relevant for the calculation of molecules adsorbed on both electrodes

\begin{tabular}{lllll}
\hline & $I_{\mathrm{pc} 1}(\mathrm{~A})$ & $Q(\mathrm{C})$ & $m(\mathrm{~mol})$ & Molecules \\
\hline Bare NEE & $1.1 \times 10^{-8}$ & $9.3 \times 10^{-8}$ & $4.8 \times 10^{-13}$ & $2.9 \times 10^{11}$ \\
AuNPs-NEE & $3.7 \times 10^{-8}$ & $3.3 \times 10^{-7}$ & $1.7 \times 10^{-12}$ & $1.0 \times 10^{12}$ \\
\hline
\end{tabular}


$10^{-3} \mathrm{~cm}^{2}$ for the NEE and $7.8 \times 10^{-3} \mathrm{~cm}^{2}$ for the AuNPsNEE.

These values can be used to estimate the roughness factor, $\rho$, defined as [22]:

$\rho=\mathrm{A}_{\mathrm{m}} / \mathrm{A}_{\mathrm{p}}$

where $A_{\mathrm{m}}$ is the "microscopic" or "true" area and $A_{\mathrm{p}}$ is the "projected" area, i.e., the area corresponding to a suitable (flat) cross-section of the metallic surface of the electrode (ensemble).
In the case of the AuNPs-NEE, the roughness factor can be evaluated as the ratio between the electrode surface measured (as described above) from the charge required for reducing the PMA adsorbed on the AuNPs-NEE and the area measured by reducing PMA adsorbed on the NEE. From the above figures, the calculated $\rho$ value is, approximately, 3.4.

Simple geometrical considerations indicate that such a roughness factor is achieved when, in average, five AuNPs are bound to the outer surface of each nanoelectrode. This seems a reasonable value, taking into account that the measured diameter for our nanoelectrodes [27] and nanoparticles are 50 and $16 \mathrm{~nm}$, respectively.
Fig. 7 Cyclic voltammograms recorded with (a) a $2 \mathrm{D} \mathrm{NEE}$ and (b) an AuNPs-NEE both functionalized with SHD1 and treated with D2-GOx, in $10 \mathrm{mM}$ PBS $(\mathrm{pH}=7)$ containing $0.1 \mathrm{mM} \mathrm{FA}^{+} \mathrm{PF}_{6}^{-}$before (full lines) and after (dashed lines) the addition of $0.05 \mathrm{M}$ glucose. Scan rate is $2 \mathrm{mVs}^{-1}$



b

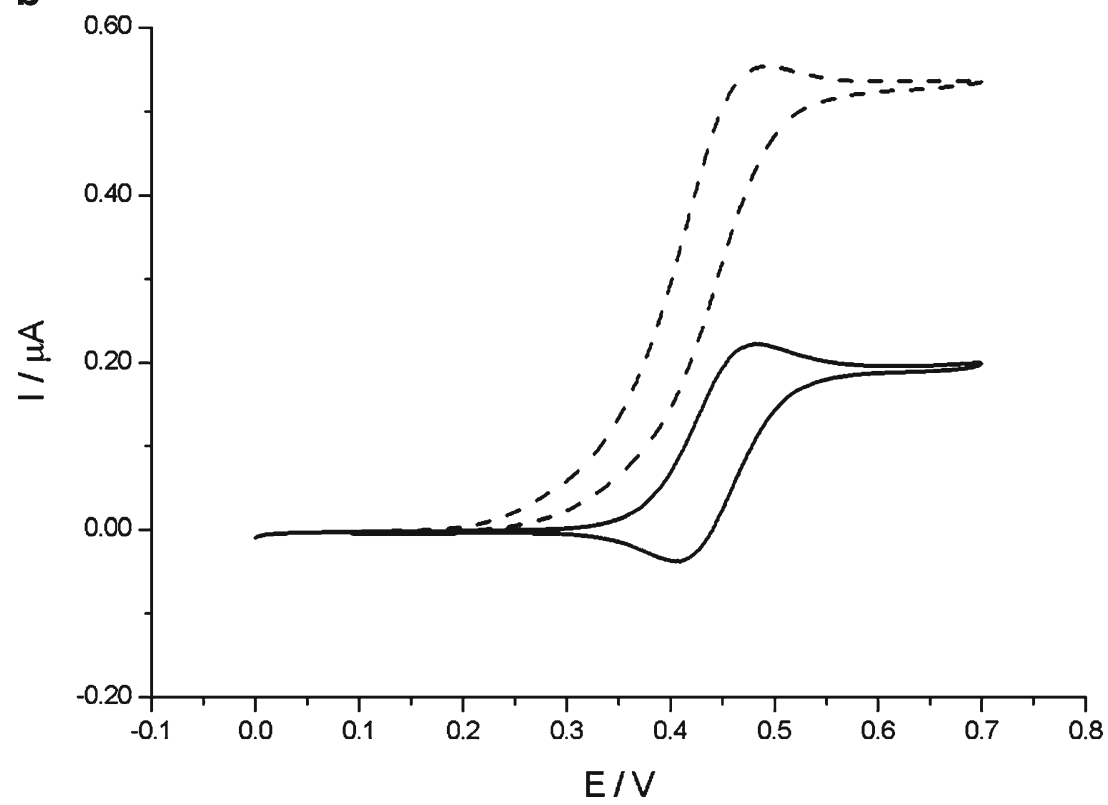


Use of AuNPs-NEEs for DNA-hybridization detection

Tests aimed at verifying the use of these devices as platforms to produce electrochemical biosensors were performed. The procedure consisted in the direct immobilization of thiolated oligonucleotides (SHD1) directly onto the metal surfaces of a 2D and a 3D NEE and the subsequent hybridization with complementary target sequences conjugated with GOx (i.e., D2-GOx, see the "Experimental" section).

A blocking step with thiols is often carried out between the immobilization of ssDNA onto the electrodic surface and the hybridization with the target. This procedure is used both, to prevent any non specific adsorption of the target labeled with enzymes and to favor a higher accessibility of the probes bound to the Au surface [28].

In a previous paper [29], the effect of 6-mercaptohexanol $(\mathrm{MCH})$ concentration on both the conformation of singlestranded DNA on AuNPs surface and the hybridization efficiency was studied. It was shown that a $\mathrm{MCH}$ concentration $>0.1 \mathrm{mM}$ caused a significant displacement of thiolated DNA from the metal surface of the nanoparticles but, although the oligonucleotides were partly removed, the capacity for hybridization was enhanced.

In our case, the introduction of the blocking step, carried out by immersion $(1 \mathrm{~h})$ of the electrode into a $1 \mathrm{mM} \mathrm{MCH}$ solution in TE buffer, $1 \mathrm{M} \mathrm{NaCl}$, did not show any effect on the electrocatalytic signals (data not shown). Note that in our experimental conditions, it is not possible to understand whether probe displacement and improved hybridization efficiency compensate each other. Anyway, since we did not observe any net change, we decided to proceed with the simplest procedure, which is avoiding the addition of $\mathrm{MCH}$. Figure 7 shows the comparison between the results obtained with the SHD1 functionalized 2D NEE (Fig. 7a) and the AuNPs-NEE (Fig. 7b) after hybridization with the GOx labeled target, in the absence (full lines) and presence of glucose (dashed lines); the measurements were performed in deoxygenated $10 \mathrm{mM}$ PBS $(\mathrm{pH}=7)$, containing $0.1 \mathrm{mM}$ $\mathrm{FA}^{+}$, added as soluble redox mediator to shuttle electrons between the nanoelectrodes and the enzyme label.

In the absence of glucose, the $\mathrm{CV}$ patterns recorded at both NEEs are characterized by the reversible one-electron oxidation of $\mathrm{FA}^{+}$, in the forward scan, which is reduced back to the initial state in the backward scan [16]. In the presence of $0.05 \mathrm{M}$ glucose (dashed lines), the curve shape changes significantly, with more dramatic changes occurring at the AuNPs-NEE: the oxidation peak increases, tending to become sigmoidally shaped, while the reduction peak disappears.

The voltammogram shape, typical for a redox-mediated electrocatalytic process, indicates that, in the presence of both the enzyme and substrate, the following electrocatalytic cycle is operative:

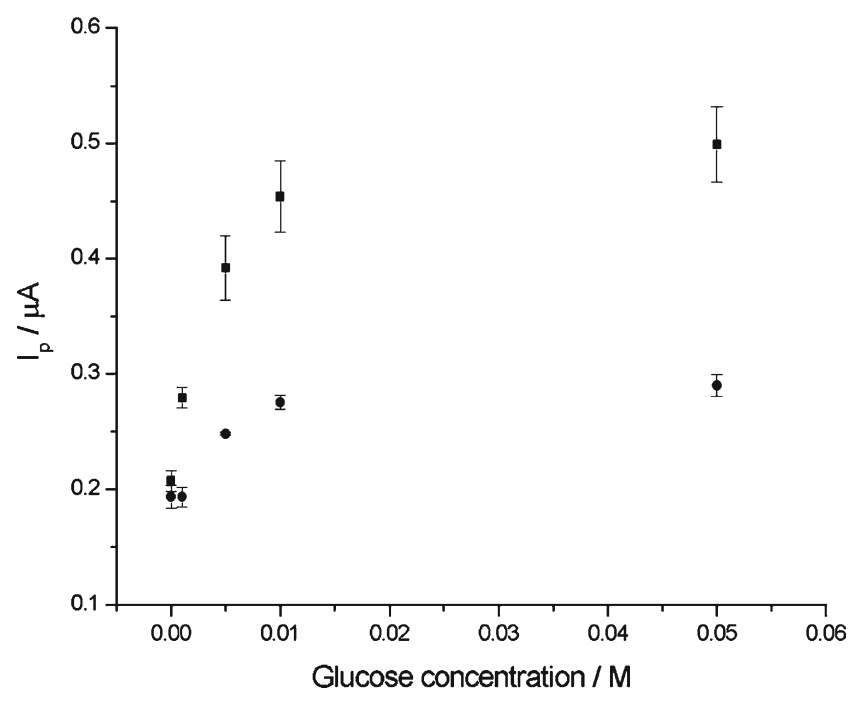

Fig. 8 Oxidation peak current values recorded with a functionalized bare NEE (circles) and a functionalized AuNPs-NEE (squares) in 10 $\mathrm{mM}$ PBS $(\mathrm{pH}=7)$ containing $0.1 \mathrm{mM} \mathrm{FA}^{+} \mathrm{PF}_{6}{ }^{-}$at increasing glucose concentrations $(0,0.001,0.005,0.010$ and $0.050 \mathrm{M})$. Scan rate is $2 \mathrm{mVs}^{-1}$. The current values presented are the average for triplicate measurements \pm standard deviation

glucose $+\mathrm{GOx}\left(\mathrm{FAD}^{+}\right) \rightarrow$ gluconolactone $+\mathrm{GOx}\left(\mathrm{FADH}_{2}\right)$

$\mathrm{FADH}_{2}+2 \mathrm{FA}^{2+} \rightarrow \mathrm{FAD}^{+}+2 \mathrm{FA}^{+}+2 \mathrm{H}^{+}$

$\mathrm{FA}^{+} \rightarrow \mathrm{FA}^{2+}+\mathrm{e}^{-}$

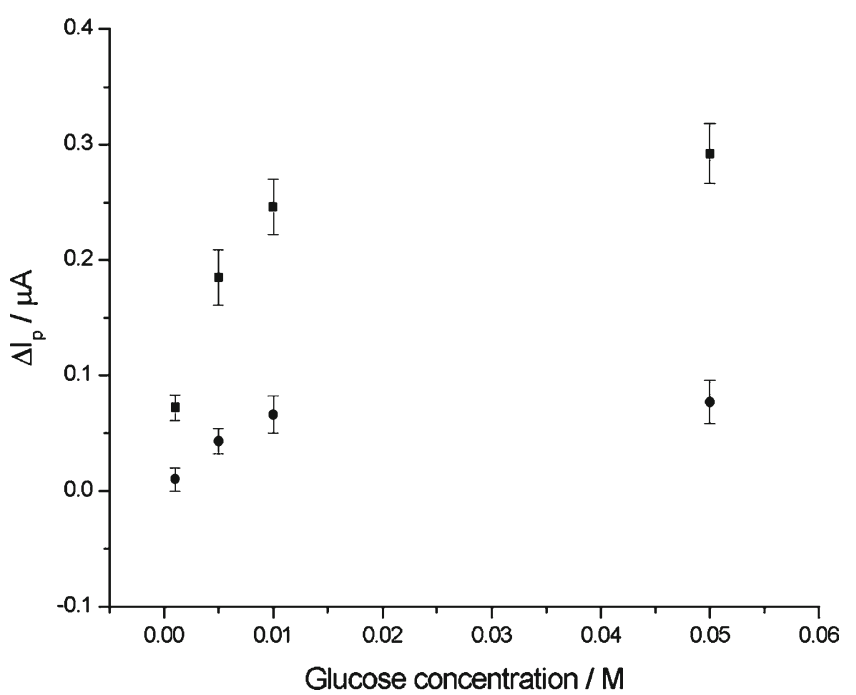

Fig. 9 Electrocatalytic current increments $\left(\Delta I_{\mathrm{p}}\right)$ recorded with a functionalized 2D NEE (circles) and a functionalized AuNPs-NEE (squares) in $10 \mathrm{mM}$ PBS $(\mathrm{pH}=7)$ containing $0.1 \mathrm{mM} \mathrm{FA}^{+} \mathrm{PF}_{6}^{-}$at increasing glucose concentrations $(0,0.001,0.005,0.010$ and $0.050 \mathrm{M})$. Scan rate is $2 \mathrm{mVs}^{-1}$. The current values presented are the average for triplicate measurements \pm standard deviation 
where the oxidation (7) takes place at the nanoelectrode/ solution interface.

It is important to note that, with NEEs modified only with the probe sequences (i.e., without any hybridization with the complementary strands conjugated with GOx), no catalytic signal was observed (data not shown).

Figure 7 clearly puts in evidence that the catalytic current is dramatically higher at the AuNPs-NEE, displaying a two times higher current increase in the presence of glucose than that recorded with the conventional 2D NEE after hybridization.

Figure 8 shows the dependence of the oxidation peak current values relative to both NEEs, at increasing glucose concentrations, for triplicate measurements. The maximum current is recorded at glucose concentrations equal or higher than $0.01 \mathrm{M}$. The comparison puts in evidence that the plateau current reached at the AuNPs-NEE is twice that of the 2D NEE.

The higher catalytic current obtained at the AuNPs-NEE is an indication of a more efficient DNA immobilization and hybridization favored by both the larger surface area and the nanostructuration of the NEE surface as a consequence of the deposition of the AuNPs. This agrees with a recent report by Kelley and coworkers [30], who demonstrated that the nanostructuration of ultramicroelectrode surfaces can improve significantly the DNA hybridization efficiency. The higher catalytic effect recorded at the AuNPs-NEE is more evident if we plot $\Delta I_{\mathrm{p}}$ vs. glucose concentration (Fig. 9). In this way, the anodic current peak value recorded at both NEEs, before the addition of glucose, is subtracted to the current measured in the presence of increasing concentrations of substrate.

For the AuNPs-NEE, the catalytic current increase is four times higher than the one at the 2D NEE.

All these results confirm that significantly higher amounts both of probe and of GOx-labeled complementary target are bound to the AuNPs-NEE as a consequence of the larger active area and nanostructuration of the surface.

\section{Conclusions}

The results of this study demonstrate that "conventional" NEEs can be successfully modified by the controlled binding of gold nanoparticles on their surface by exploiting the binding capabilities of bi-functional thiols such as cysteamine. Such a modification can be particularly useful in order to fabricate AuNPs-NEEs characterized by increased sensitivity to be used for advanced sensing or biosensing applications. According to the presented procedure, no physical or chemical etching of the polycarbonate of the NEEs is required to obtain $3 \mathrm{D}$ structures. In addition to simplifying the experimental procedure to obtain the $3 \mathrm{D}$
NEEs, avoiding the etching means avoiding the excessive (and difficult to control) increase of the active area (and double-layer charging current) often associated to the etching itself [7]. The two most widely used etching procedures, i.e., $\mathrm{O}_{2}$ plasma and chemical etching with dichloromethane, cause indeed the loss of the sealing between the PC membrane and the gold nanofibers [7] making the increase of the active area difficult to control.

It is also important to note that these electrodes maintain an "ensemble" structure, consequently keeping all the advantages typical of such a geometry. Moreover, our results show that both the AuNPs and the SAM of cysteamine do not block the electron transfer between electroactive analytes (in this case the redox mediator $\mathrm{FA}^{+}$) and the underlying nanoelectrodes.

Finally, the experiments reported here put in evidence that AuNPs-NEEs, after functionalization with biorecognition layers, can be used as efficient electrochemical biosensors with improved sensitivity with respect to 2D NEEs.

Acknowledgments This work was supported by MIUR (Rome), project: PRIN 2008MWHCP2 and by the Cross-Border Cooperation Italy-Slovenia Programme 2007-2013 - Strategic Project TRANS2CARE. We thank Dr. Loredana Casalis (ELETTRA, Sincrotrone Trieste, Scuola Internazionale Superiore di Studi Avanzati) for helpful discussion and suggestions, Dr. Davide Cristofori (University $\mathrm{Ca}$ ' Foscari) for TEM measurements and Dr. Ljiljana Fruk (KIT-Karlsruhe Institute of Technology) for oligonucleotide stock solutions, and Dania Kendziora (KIT - Karlsruhe Institute of Technology) for D2GOx conjugation.

\section{References}

1. Menon VP, Martin CR (1995) Anal Chem 67:1920-1928

2. Moretto LM, Panero S, Scrosati B, Ugo P (2009) In: Lin Y, Nalwa HS (eds) Handbook of electrochemical nanotechnology, vol 1. ASP, Stevenson Ranch

3. Ugo P, Moretto LM, Vezzà F (2002) ChemPhysChem 3:917-925

4. Yu S, Li N, Wharton J, Martin CR (2003) Nano Lett 3:815-818

5. Krishnamoorthy K, Zoski CG (2005) Anal Chem 77:5068-5071

6. Gasparac R, Taft BJ, Lapierre-Devlin MA, Lazareck AD, Xu JM, Kelley SO (2004) J Am Chem Soc 126:12270-12271

7. De Leo M, Kuhn A, Ugo P (2007) Electroanalysis 19:227-236

8. Dyne J, Lin Y-S, Lai LMH, Ginges JZ, Luais E, Peterson JR, Goon IY, Amal R, Gooding JJ (2010) ChemPhysChem 11:2807-2813

9. Wang J (2002) Anal Chim Acta 469:63-71

10. Silvestrini M, Fruk Lj, Ugo P (2012) Biosens Bioelectron. doi:10.1016/j.bios.2012.07.041

11. Abys JA, Maisano JJ (2000) US Patent $N^{\circ} 6,126,807$

12. Fruk LJ, Müller J, Weber G, Narvaez A, Dominguez E, Niemeyer CM (2007) Chem Eur J 13(18):5223-5231

13. Shein JB, Lai LMH, Eggers PK, Paddon-Row MN, Gooding JJ (2009) Langmuir 25:11121-11128

14. Haiss W, Thanh NTK, Aveyard J, Fernig DG (2007) Anal Chem 79:4215-4221

15. Silvestrini M, Schiavuta P, Scopece P, Pecchielan G, Moretto LM, Ugo P (2011) Electrochim Acta 56:7718-7724

16. Ugo P, Moretto LM (2007) In: Zoski CG (ed) Handbook of electrochemistry, chapter 16.2. Elsevier, Amsterdam 
17. Yang W, Wang J, Zhao S, Sun Y, Sun C (2006) Electrochem Commun 8:665-672

18. Cai H, Xu C, He P, Fang Y (2001) J Electroanal Chem 510:78-85

19. Leff DV, Brandt L, Heath JR (1996) Langmuir 12:4723-4730

20. Hoft RC, Ford MJ, McDonagh AM, Cortie MB (2007) J Phys Chem C 111:13886-13891

21. Peterson AW, Heaton RJ, Georgiadis RM (2001) Nucleic Acids Res 29(24):5163-5168

22. Bard AJ, Faulkner LR (2001) Electrochemical methods. Fundamentals and applications, 2nd edn. Wiley, New York

23. Wang Y, Weinstock IA (2010) Dalton Trans 39:6143-6152
24. Kaba MS, Song IK, Barteau MA (1996) J Phys Chem 100:1957719581

25. Zhang G, He T, Ma Y, Chen Z, Yang W, Yao J (2003) Phys Chem Chem Phys 5:2751-2753

26. Martel D, Sojic N, Kuhn A (2002) J Chem Ed 79:349-352

27. Ugo P, Pepe N, Moretto LM, Battagliarin M (2003) J Electroanal Chem 560:51-58

28. Herne TM, Tarlov MJ (1997) J Am Chem Soc 119:8916-8920

29. Park S, Brown KA, Hamad-Schifferli K (2004) Nano Lett 4 (10):1925-1929

30. Bin X, Sargent EH, Kelley SO (2010) Anal Chem 82:5928-5921 